\title{
Estructura interna y fiabilidad de la escala Percepción del Alumnado sobre el Clima Escolar (PACE-33)
}

\section{Internal consistency and reliability of the students' perception of school climate scale (PACE-33)}

\author{
Naiara Escalante Mateos ${ }^{1}$, Arantza Fernández-Zabala ${ }^{2}$, Eider Goñi Palacios ${ }^{3}$, Iker Izar-de-la-Fuente Díaz- \\ de-Cerio $^{4}$ \\ ${ }^{1}$ Facultad de Educación y Deporte Universidad del País Vasco (UPV/EHU) naiara.escalante@ehu.eus \\ ${ }^{2}$ Universidad del País Vasco (UPV/EHU) arantza.fernandez@ehu.eus \\ ${ }^{3}$ Universidad del País Vasco (UPV/EHU) eider.goni@ehu.eus \\ ${ }^{4}$ Universidad del País Vasco (UPV/EHU) iker.izardelafuente@ehu.eus
}

Recibido: 8/9/2020

Aceptado: 11/12/2020

Copyright (C)

Facultad de CC. de la Educación y Deporte. Universidad de Vigo

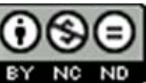

Dirección de contacto:

Naiara Escalante Mateos

Facultad de Educación y Deporte

Centro de Investigación Mikaela Portilla

(Despacho 1.9)

01006

VITORIA-GASTEIZ (ESPAÑA)

\section{Resumen}

La multitud de beneficios que comporta al alumnado un clima escolar positivo pone de manifiesto la importancia de evaluar este constructo de manera adecuada. Sin embargo, aún no se dispone de ninguna medida válida y fiable ajustada al contexto académico español que evalúe los aspectos más relevantes del clima escolar. Para superar esta limitación, se diseñó la escala Percepción del Alumnado sobre el Clima Escolar (PACE-33), instrumento que evalúa el clima escolar percibido en alumnado adolescente. El objetivo de este trabajo es corroborar mediante el análisis factorial confirmatorio la estructura del PACE-33 encontrada en el estudio exploratorio previo de nueve factores, además de analizar su fiabilidad de consistencia interna. Participaron en la investigación 654 estudiantes $\left(M_{\text {edad }}=15,41\right.$ años, $\left.D T=1,76\right)$ de ocho centros escolares de la Comunidad Autónoma del País Vasco. El modelo de nueve factores correlacionados propuesto presenta un ajuste óptimo (RMSEA= ,035 $(, 031$ - ,039); NNFI = ,919; NFI= ,956; CFI = ,962), además de índices de fiabilidad de consistencia interna apropiados. Se concluye, por tanto, que la escala PACE-33 permite conocer de manera válida y fiable la percepción del alumnado acerca de los principales aspectos que favorecen el clima escolar en la adolescencia.

\section{Palabras clave}

Clima Escolar, Instrumento de Medida, Análisis Factorial Confirmatorio, Fiabilidad, Adolescencia

\section{Abstract}

The multitude of benefits that a positive school climate brings to the students shows the importance of evaluating this construct appropriately. However, there is still no valid and reliable measure adjusted to the Spanish academic context that evaluates 
the most relevant aspects of the school climate. To overcome this limitation, the students' perception of school climate scale (PACE-33) was designed, an instrument that evaluates the perceived school climate in adolescent students. The aim of this work is to corroborate the nine-factor structure of the PACE-33 found in the previous exploratory study by means of confirmatory factor analysis, in addition to analyzing its internal consistency reliability. A total of 654 students $\left(M_{\text {age }}=15,41\right.$ years, $S D=$ 1,76) from eight schools in the Basque Country participated in the research. The model of nine correlated factors proposed presents an optimal fit (RMSEA $=, 035_{(, 031}$ ,039); NNFI = ,919; $\mathrm{NFI}=$,956; CFI = ,962), as well as appropriate internal consistency reliability indices. In conclusion, the PACE-33 scale allows us to know in a valid and reliable way the perception of the students about the main aspects that favour the school climate in adolescence.

\section{Key Words}

School Climate, Measurement Instrument, Confirmatory Factor Analysis, Reliability, Adolescence

\section{INTRODUCCIÓN}

El objetivo principal de todo centro educativo es promover el aprendizaje y el desarrollo del alumnado (Alonso-Tapia et al., 2020). En este complejo proceso, el clima escolar es esencial dado que está considerado como una de las piezas clave de la eficacia, calidad y mejora del centro escolar (Aron, Milicic y Armijo, 2012). Numerosos estudios muestran un fuerte vínculo entre el clima escolar positivo, esto es, un clima caracterizado por la valoración y aceptación del alumnado en un ambiente basado en el apoyo, las claras expectativas conductuales, la legitimidad de las normas, la comunicación y el trabajo colaborativo, y los resultados académicos, conductuales y psicológicos del alumnado (Maxwell, Reynolds, Lee, Subasic y Bromhead 2017; Quijada, Ruiz, Huertas y Alonso-Tapia, 2020). Además, está comprobado que esos resultados se mantienen en estudiantes de diferentes niveles, orígenes y culturas (Jia et al., 2009). El clima escolar positivo se ha asociado, entre otros muchos, con mejores resultados académicos (Alonso-Tapia et al., 2020; Daily, Mann, Kristjansson, Smith y Zullig 2019; López-González y Oriol, 2016), una menor prevalencia del acoso escolar (Farina, 2019; Hong, Espelage y Lee, 2018) y de la delincuencia del alumnado (Gottfredson Gottfredson, Payne, y Gottfredson, 2005; Patton et al., 2006), una mayor cohesión de grupo (Daniels, 2016), un compromiso con la escuela más alto (Bosworth y Judkins, 2014; Orpinas y Raczynski, 2016), una mayor satisfacción con la vida (Aldridge et al., 2016) y un mejor bienestar psicológico (Bondía, Martínez, Melendro y Mora, 2019; Newland, DeCino, Mourlam y Strous 2019; Varela et al., 2019). Por todo esto, y debido a la gran capacidad de cambio que posee el clima escolar, actualmente hay abundantes iniciativas de reforma escolar que se centran en mejorar este constructo (Alonso-Tapia et al., 2020; Durlak, Weissberg, Dymnicki, Taylor y Schellinger, 2011) y son numerosos los estudios que subrayan la importancia de evaluarlo adecuadamente con el objetivo de mejorar la calidad de las instituciones educativas (Quijada et al., 2020; Sun y Royal, 2017). Es más, autores como Bondía et al. (2019), Herrera y Ballesteros (2014) y Wang y Degol (2016) afirman que la evaluación del clima escolar es una vía de trabajo prioritaria y fundamental. Sin embargo, es un constructo difícil de abordar y de evaluar debido principalmente a que se trata de una estructura 
multidimensional que ha sido aprehendida desde diferentes perspectivas teóricas y metodológicas (Maxwell et al., 2017). La existencia de divergencias en la interpretación del concepto clima escolar así como en la enunciación de las dimensiones que lo componen, ha implicado que hayan sido numerosos y muy diversos los instrumentos creados, especialmente en los países anglosajones, para la evaluación de la percepción del alumnado sobre dicho constructo (Cohen, McCabe, Michelli y Pickeral, 2009; Escalante, Fernández-Zabala, Goñi e Izar-de-la-Fuente, 2020). Sin embargo, tal y como comprobaron Escalante, Fernández-Zabala, Goñi, e Izar-de-la-Fuente (2020) en su estudio tras revisar más de 50 instrumentos de medida diseñados para la evaluación del clima escolar, hoy en día, aún, no está claramente delimitada la estructura interna de este constructo y no se cuenta con ningún instrumento en castellano válido y fiable que mida de forma equilibrada las dimensiones específicas de los cuatro dominios principales que la mayoría de los estudios respaldan como componentes del clima escolar, esto es, seguridad, relaciones, aspectos ambientales-estructurales y enseñanzaaprendizaje (Cohen et al., 2009; National School Climate Center, 2014). Para tratar de solventar estas limitaciones, se diseñó la escala Percepción del Alumnado sobre el Clima Escolar (PACE-33), que posibilita la evaluación de los aspectos específicos más relevantes de los cuatro dominios del clima escolar mencionados anteriormente. En el análisis factorial exploratorio llevado a cabo en el estudio piloto de esta escala (Escalante, Fernández-Zabala, Goñi e Izar-de-la-Fuente, 2020) se extrae una solución compuesta por nueve factores: seguridad física, normas, relación alumnadoprofesorado, relación entre iguales, cohesión de grupo, aspectos ambientalesestructurales, capacidad de motivación del profesorado, expectativas del profesorado y recursos metodológicos. El objetivo de este trabajo es corroborar mediante el análisis factorial confirmatorio dicha estructura de nueve factores y analizar su fiabilidad de consistencia interna en orden a completar su validación. Se opta por someter a prueba la estructura de nueve factores correlacionados debido a que, por un lado, diversos autores que analizan la estructura interna de escalas que tienen por objetivo evaluar la percepción del alumnado (Hung, Luebbe y Flaspohler, 2015; Muñoz et al., 2018) o del profesorado (Quijada et al., 2020) sobre el clima escolar hallan una estructura de factores correlacionados, y por otro lado, autores como Shindler, Jones, Williams, Taylor y Cardenas (2016) afirman que las dimensiones del clima escolar están altamente correlacionadas entre sí, lo que indica que son fuertemente interdependientes.

\section{MÉTODO}

\subsection{Participantes}

Inicialmente participaron un total de 683 estudiantes de Educación Secundaria Obligatoria (ESO) y Bachillerato provenientes de ocho centros escolares de la Comunidad Autónoma del País Vasco, seis públicos y dos concertados. No obstante, debido a la eliminación de los sujetos que no habían respondido a más del $10 \%$ de los ítems y de los outliers (sujetos con respuestas muy extremas o inconsistentes), la muestra finalmente quedó compuesta por 654 estudiantes de $1^{\mathrm{o}}$ de Educación Secundaria Obligatoria (ESO) a $2^{\circ}$ de Bachillerato. El rango de edad fue de 12 a 19 años $\left(M_{\text {edad }}=15,41\right.$ años, $\left.D T=1,76\right)$. Del conjunto de la muestra $341(52,1 \%)$ eran hombres 
y $313(47,9 \%)$ mujeres, de los cuales $226(34,6 \%)$ cursaban $1^{\circ}$ ciclo de la ESO, 250 $(38,2 \%) 2^{\circ}$ ciclo de la ESO y 178 (27,2\%) Bachillerato (Tabla 1). La selección de la muestra se llevó a cabo de forma incidental.

\begin{tabular}{ccccc}
\hline & \multicolumn{5}{c}{ Ciclo escolar } \\
\hline Sexo & $1^{\circ}$ ciclo ESO & $2^{\circ}$ ciclo ESO & Bachillerato & Total \\
\hline Mujer & $108(16,5 \%)$ & $128(19,6 \%)$ & $77(11,8 \%)$ & $313(47,9 \%)$ \\
\hline Hombre & $118(18,1 \%)$ & $122(18,6 \%)$ & $101(15,4 \%)$ & $341(52,1 \%)$ \\
\hline Total & $226(34,6 \%)$ & $250(38,2 \%)$ & $178(27,2 \%)$ & $654(100 \%)$ \\
\hline
\end{tabular}

Tabla 1. Frecuencias y porcentajes de hombres y mujeres en cada ciclo escolar

\subsection{Instrumentos de medida}

El clima escolar percibido se evaluó con la escala PACE-33 (Escalante, FernándezZabala, Goñi e Izar-de-la-Fuente, 2020) (Anexo 1). Se trata de un autoinforme que cuenta con un formato de respuesta en escala Likert de cinco grados donde $1=$ totalmente en desacuerdo y $5=$ Totalmente de acuerdo. Contiene un total de 33 ítems, 29 directos y 4 inversos, que se organizan en nueve subescalas: (1) seguridad física; (2) normas; (3) relación alumnado-profesorado; (4) relación entre iguales; (5) cohesión de grupo; (6) aspectos ambientales-estructurales; (7) capacidad de motivación del profesorado; (8) expectativas del profesorado; y (9) recursos metodológicos. En la evaluación psicométrica previa se constató que estas nueve subescalas tienen una buena fiabilidad de la consistencia interna: seguridad física $(\alpha=, 805)$, normas $(\alpha=, 797)$, relación alumnado-profesorado $(\alpha=, 852)$, relación entre iguales $(\alpha=, 890)$, cohesión de grupo $(\alpha=, 868)$, aspectos ambientales-estructurales $(\alpha=, 753)$, capacidad de motivación del profesorado $(\alpha=, 927)$, expectativas del profesorado $(\alpha=, 847)$ y recursos metodológicos $(\alpha=, 874)$.

\subsection{Procedimiento}

En primer lugar, se mantuvo una reunión con el equipo directivo de cada uno de los centros educativos, en la que se les explicó en detalle la finalidad de esta investigación y se les solicitó su participación en la misma. Tras obtener la aprobación de colaboración de la dirección de los centros, se les entregó el consentimiento informado que debían firmar tanto los progenitores o los tutores legales como el propio alumnado participante. Una vez obtenidos dichos consentimientos informados, se concretaron unas fechas y horarios con la jefatura de estudios de los centros para aplicar entre el alumnado la escala PACE-33. Esta escala la administraron dos de las investigadoras de este trabajo en horario lectivo y de manera simultánea a todo el alumnado integrante de una misma aula con el fin de asegurar la uniformidad en la aplicación. Las investigadoras dieron las explicaciones oportunas para que el alumnado cumplimentase la escala de manera adecuada. Asimismo, subrayaron la importancia de responder con sinceridad a los distintos ítems, insistiendo en el carácter confidencial y voluntario de la prueba, en orden a reducir la tendencia de deseabilidad social en las respuestas. Para evitar amenazas a la validez de resultados, además, siguieron el criterio de ciego único, evitando que las personas participantes conociesen la finalidad de la investigación. 


\subsection{Análisis de datos}

En esta investigación se utilizaron dos programas estadísticos: el SPSS v.25 (IBM Corporation, 2017) y el EQS v.6.2 (Bentler, 2006). Se hizo uso del programa estadístico SPSS v.25 para los análisis preliminares de los datos, y del EQS v.6.2, por un lado, en los análisis previos para evaluar el coeficiente estandarizado de curtosis de Mardia, y por otro, para el análisis factorial confirmatorio realizado posteriormente.

Como parte de los análisis preliminares, primeramente, se eliminaron los individuos que no habían respondido a más del $10 \%$ del total de ítems y/o los que presentaron patrones de respuesta extraños o inconsistentes, esto es, 26 individuos. Seguidamente, tras comprobar que el número de valores perdidos no excedía del $5 \%$ del total de los datos analizados y que el patrón de datos perdidos era aleatorio, se imputaron dichos valores mediante el método de estimación de tendencia lineal en el punto. A continuación, se identificaron y eliminaron tres casos con valores atípicos mediante el procedimiento de detección de anomalías. Por último, antes de proceder al análisis factorial confirmatorio, se examinó el ajuste de los datos a una distribución normal multivariada. A este respecto, Bentler y Wu (2002) afirman que el coeficiente estandarizado de curtosis de Mardia debe presentar un valor inferior a 3 para poder asumir el supuesto de normalidad multivariante en el conjunto de datos.

Para establecer el ajuste del modelo de nueve factores correlacionados, se llevó a cabo un análisis factorial confirmatorio a través del método de estimación robusto de máxima verosimilitud, puesto que el coeficiente estandarizado de Mardia indicó que la distribución multivariada de los datos no era normal (Bentler y Wu, 2002). El ajuste del modelo se valoró empleando los siguientes índices robustos: Satorra-Bentler chicuadrado $\left(\mathrm{SB} \chi^{2}\right)$; razón entre la Satorra-Bentler chi-cuadrado y los grados de libertad $\left(\mathrm{SB} \chi^{2} / g l\right)$, índice que debe obtener un valor próximo al punto de corte de 2 que señalan Tabachnick y Fidell (2007) para considerarse adecuado; raíz cuadrada del error medio cuadrático (RMSEA) y su respectivo intervalo de confianza del $90 \%$, cuyo valor inferior a ,06 es indicativo de un ajuste óptimo (Kline, 2015); índice de ajuste comparativo (CFI), índice de ajuste no normalizado (NNFI) e índice de ajuste normalizado (NFI), en los que valores superiores a ,90 son representativos de un ajuste adecuado (Kline, 2015).

La fiabilidad de la consistencia interna de la subescalas se examinó mediante el alpha de Cronbach y los coeficientes de fiabilidad compuesta omega de McDonald y rho de Raykov. Se consideran adecuados los valores superiores a ,70 en estos tres índices (Hair, Babin, Anderson y Black, 2018). Además, se estimó la Varianza Media Extraída (VME) como indicador de la validez de la estructura interna de la escala. En este último caso, se recomiendan puntuaciones próximas al punto de corte establecido de ,50 (Hair et al., 2018). Bettencourt (2004), sin embargo, matiza dicho criterio y afirma que valores de VME inferiores a ,50 pueden considerarse aceptables, siempre que presenten coeficientes de fiabilidad compuesta superiores a ,70 y correlaciones ítem-total que superen el, 40 . 


\section{RESULTADOS}

\subsection{Estructura interna de la escala PACE-33}

El modelo de nueve factores correlacionados propuesto (Figura 1) muestra un ajuste adecuado: $\mathrm{SB} \chi^{2}(459)=833,788 p<, 05 ; \mathrm{SB} \chi^{2} / g l=1,82 ; \operatorname{RMSEA}_{(90 \%)}=, 035_{(, 031,039)}$; $\mathrm{NFI}=$,919; NNFI = 956; CFI = ,962. En este modelo la distribución de frecuencias de los residuos estandarizados pone de manifiesto que el 95,55\% de los residuos presentan valores centrales entre $-0,1$ y 0,1 . En lo que respecta a los coeficientes no estandarizados y los errores típicos, se verifica que todos muestran valores razonables y estadísticamente significativos. En cuanto a los coeficientes de regresión estandarizados, todos los ítems logran cargas superiores a ,40. Los coeficientes de regresión entre los ítems y los factores oscilan entre ,461 y ,920 (Tabla 2).

En lo referente a las relaciones entre los factores latentes, las covarianzas están comprendidas entre .073 y .632 siendo los factores relación alumnado-profesorado y capacidad de motivación del profesorado (F3-F7) los que se asocian en mayor medida. En cambio, los factores que menor grado de asociación muestran son seguridad física y normas (F1-F2) y relación entre iguales y capacidad de motivación del profesorado (F4-F7).

\subsection{Fiabilidad de la consistencia interna y VME de las subescalas del PACE-33}

En la Tabla 3 se presentan los diferentes coeficientes de fiabilidad de consistencia interna y la VME de las nueve subescalas del PACE-33.

En cuanto a la fiabilidad de la consistencia interna, todas las subescalas presentan valores adecuados, esto es, superiores a ,70. Los valores están comprendidos entre ,706 y ,921 para el alpha de Cronbach, entre ,715 y ,922 para el omega de McDonald y entre ,723 y ,922 para el rho de Raykov.

Respecto a la VME, todas las subescalas presentan valores adecuados superiores a ,50, a excepción de la subescala aspectos ambientales-estructurales que presenta un valor inferior $(\mathrm{VME}=, 404)$. De todas formas, de acuerdo a lo afirmado por Bettencourt (2004), dicho valor puede considerarse aceptable ya que la subescala muestra coeficientes de fiabilidad compuesta razonables $(\omega=, 722$ y $\rho=, 724)$ y se verifica que las correlaciones ítem-subescala corregidas son superiores a , 40 . 


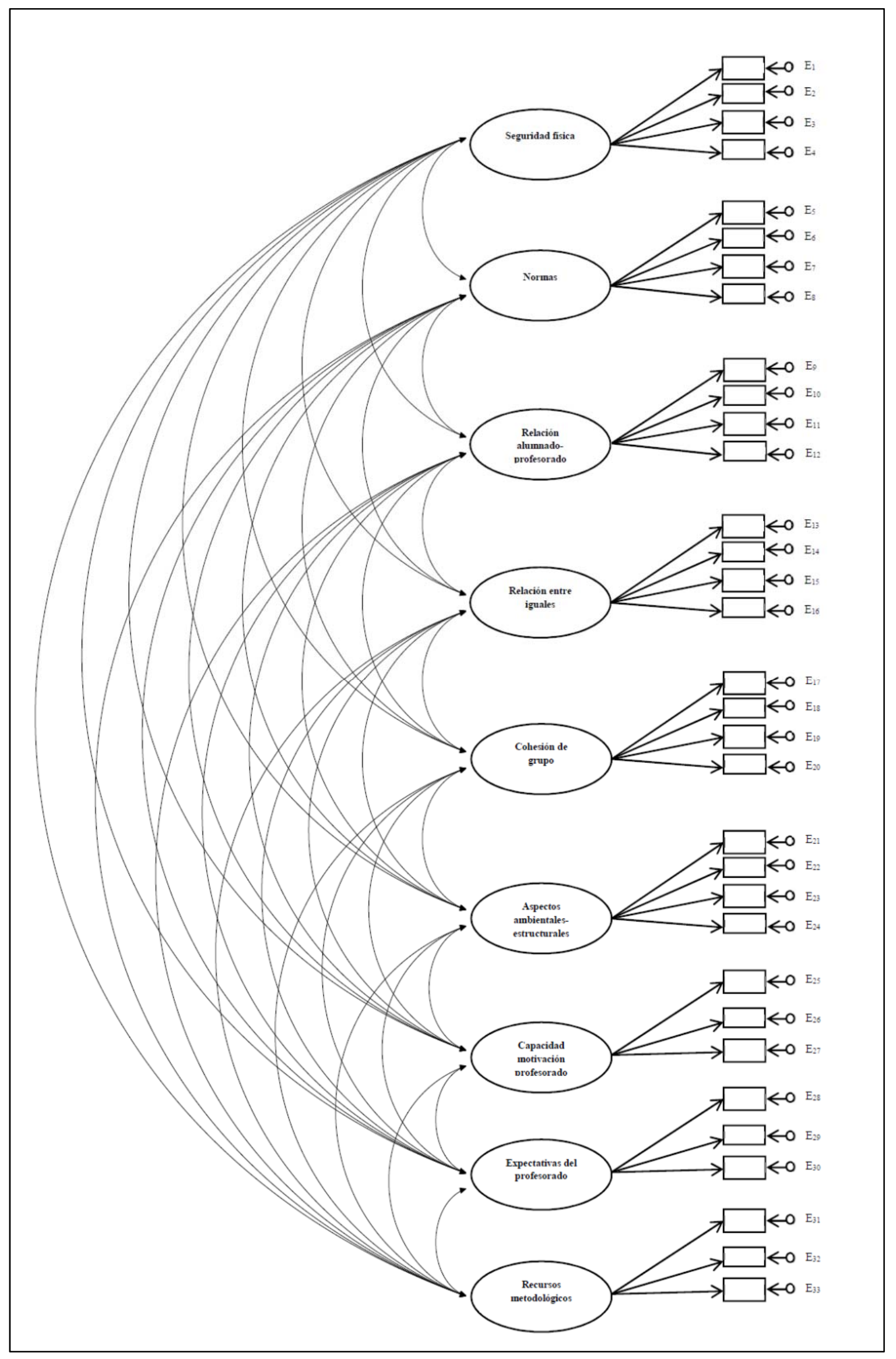

Figura 1. Modelo de nueve factores correlacionados 


\begin{tabular}{|c|c|c|c|c|c|c|c|c|c|}
\hline & $\mathrm{F} 1$ & $\mathrm{~F} 2$ & F3 & $\mathrm{F} 4$ & F5 & F6 & F7 & F8 & F9 \\
\hline PACE01 & ,801 & & & & & & & & \\
\hline PACE02 &, 824 & & & & & & & & \\
\hline PACE03 & ,658 & & & & & & & & \\
\hline PACE04 & ,825 & & & & & & & & \\
\hline PACE05 & &, 754 & & & & & & & \\
\hline PACE06 & & ,792 & & & & & & & \\
\hline PACE07 & & 805 & & & & & & & \\
\hline PACE08 & &, 764 & & & & & & & \\
\hline PACE09 & & &, 801 & & & & & & \\
\hline PACE10 & & &, 783 & & & & & & \\
\hline PACE11 & & &, 731 & & & & & & \\
\hline PACE12 & & & ,739 & & & & & & \\
\hline PACE13 & & & & ,758 & & & & & \\
\hline PACE14 & & & & ,801 & & & & & \\
\hline PACE15 & & & & ,798 & & & & & \\
\hline PACE16 & & & &, 860 & & & & & \\
\hline PACE17 & & & & &, 769 & & & & \\
\hline PACE18 & & & & & 843 & & & & \\
\hline PACE19 & & & & &, 776 & & & & \\
\hline PACE20 & & & & &, 755 & & & & \\
\hline PACE21 & & & & & & ,566 & & & \\
\hline PACE22 & & & & & & ,461 & & & \\
\hline PACE23 & & & & & &, 734 & & & \\
\hline PACE24 & & & & & &, 737 & & & \\
\hline PACE25 & & & & & & & 865 & & \\
\hline PACE26 & & & & & & & ,920 & & \\
\hline PACE27 & & & & & & & 894 & & \\
\hline
\end{tabular}

Tabla 2. Coeficientes de regresión ítem-factor 


\begin{tabular}{|c|c|c|c|c|c|c|c|c|c|}
\hline & $\mathrm{F} 1$ & $\mathrm{~F} 2$ & F3 & $\mathrm{F} 4$ & F5 & F6 & F7 & F8 & F9 \\
\hline PACE28 &, 752 & & & & & & & & \\
\hline PACE29 &, 856 & & & & & & & & \\
\hline PACE30 &, 867 & & & & & & & & \\
\hline PACE31 & &, 800 & & & & & & & \\
\hline PACE32 & & ,836 & & & & & & & \\
\hline PACE33 & & 878 & & & & & & & \\
\hline
\end{tabular}

Nota. F1 = seguridad física; F2 = normas; F3= relación alumnado-profesorado; F4 = relación entre iguales; F5 = cohesión de grupo; F6 = aspectos ambientales-estructurales; F7 = capacidad de motivación del profesorado; F8 = expectativas del profesorado; F9 = recursos metodológicos.

Tabla 2. Coeficientes de regresión ítem-factor (Continuación)

\begin{tabular}{ccccc}
\hline & $\alpha$ & $\omega$ & $\rho$ & VME \\
\hline Seguridad física &, 851 &, 858 &, 861 &, 609 \\
\hline Normas &, 860 &, 860 &, 861 &, 607 \\
\hline Rela. al-pro &, 846 &, 848 &, 849 &, 584 \\
\hline Rela. iguales &, 879 &, 880 &, 880 &, 648 \\
\hline Cohesión &, 864 &, 866 &, 866 &, 619 \\
\hline Ambiente &, 706 &, 715 &, 723 &, 404 \\
\hline Motivación &, 921 &, 922 &, 922 &, 798 \\
\hline Expectativas &, 862 &, 864 &, 866 &, 683 \\
\hline Recursos &, 875 &, 876 &, 877 &, 703 \\
\hline
\end{tabular}

Nota. $\alpha$ : Alpha de Cronbach; $\omega$ : Omega de Mcdonald; $\rho$ : Rho de Raykov; VME: Varianza Media Extraída; Rela. al-pro: Relación alumnado profesorado; Rela. iguales: Relación entre iguales; Cohesión: Cohesión de grupo; Ambiente: Aspectos ambientales-estructurales; Motivación: Capacidad de motivación del profesorado; Expectativas: Expectativas del profesorado; Recursos: Recursos metodológicos.

Tabla 3. Coeficientes de fiabilidad y VME de las subescalas del PACE-33

\section{DISCUSIÓN}

Dado que está comprobado que son abundantes los beneficios que reporta un clima escolar positivo al alumnado (Maxwell et al., 2017; Quijada et al, 2020), son numerosos los estudios que subrayan la necesidad de evaluar este constructo adecuadamente. Sin embargo, aún no se conoce con precisión la estructura interna de esta variable y no se dispone de un instrumento de medida válido y fiable en castellano que posibilite la adecuada evaluación de la percepción del alumnado adolescente sobre las dimensiones más relevantes del clima escolar (Escalante, Fernández-Zabala, Goñi e Izar-de-laFuente, 2020; Escalante, Goñi, Fernández-Zabala e Izar-de-la-Fuente, 2020). Esta investigación surge precisamente con la intención de suplir esta carencia, y para ello, aporta una escala validada en el contexto español que evalúa los aspectos más destacados del clima escolar de forma fiable. Los resultados obtenidos en este estudio constatan que el PACE-33 permite medir de manera eficaz y con precisión la percepción del alumnado adolescente español acerca de aspectos relativos a la seguridad 
física, las normas, la relación entre el profesorado y el alumnado, la relación entre los iguales, la cohesión de grupo, los aspectos ambientales-estructurales, la capacidad de motivación del alumnado, las expectativas del profesorado y los recursos metodológicos.

En lo que respecta a la estructura interna del PACE-33, los resultados constatan que el modelo de nueve factores correlacionados propuesto presenta un ajuste óptimo, y que, por tanto, reproduce de manera adecuada los datos observados. Esta estructura evidenciaría la disposición de las dimensiones que conforman el clima escolar. Este resultado es congruente con el obtenido en estudios previos (Hung et al., 2014; Muñoz et al., 2018), que si bien analizan la estructura interna de escalas para la evaluación de la percepción del alumnado sobre el clima escolar compuestas por subescalas distintas a las del PACE-33, confirman también una estructura compuesta por distintos factores correlacionados. En la misma línea, Quijada et al. (2020) constatan una estructura de factores correlacionados relativa a la percepción que posee el profesorado acerca de algunas de las dimensiones más destacadas del clima escolar.

En lo referente a la fiabilidad del PACE-33, los coeficientes de consistencia interna logrados en este estudio confirman la fiabilidad de las puntuaciones obtenidas en todas las subescalas del PACE-33, lo que es indicativo de que todas miden con precisión las dimensiones más destacadas del clima escolar percibido. Esto diferencia a la escala PACE-33 de otras como La Escala Breve de Clima de Clase (EBCC) de LópezGonzález y Bisquerra (2013) o la Escala de Clima Social Escolar (ECLIS) de Aron et al. (2012), en las que no todas las subescalas que las componen alcanzan el ,70 exigido. Además, los resultados obtenidos demuestran que la mayoría de las subescalas del PACE-33 tienen valores superiores a ,50 en la VME, lo que mostraría que al menos la mitad de la variación en sus ítems se debe al factor latente al que pertenecen (Hair et al., 2018), y confiere validez a la estructura interna de la escala.

En cualquier caso, hay que señalar que este estudio no está exento de limitaciones. La limitación principal de este trabajo radica en que, a pesar de que se ha contado con una muestra heterogénea y representativa de la población, el alumnado participante pertenece a una sola comunidad autónoma y ha sido seleccionado de forma incidental, por lo tanto, sería pertinente que futuros estudios ratificasen los resultados obtenidos en este trabajo con una muestra más amplia y aleatoria que abarcase alumnado de otras comunidades autónomas.

En cualquier caso, este estudio ofrece evidencias de la estructura interna y de la fiabilidad de la escala PACE-33 en población adolescente española y supone un avance importante en la delimitación de la estructura interna del clima escolar, una de las variables más importantes de la eficacia, calidad y mejora de la institución educativa (Aron et al., 2012). Disponer de una medida válida y fiable como es el PACE-33 para la evaluación de la percepción del alumnado sobre los aspectos más importantes del clima escolar permitirá a los centros educativos tomar decisiones basadas en datos y optimizar los recursos con los que cuentan, lo cual es esencial para que puedan brindar una respuesta educativa adecuada. 


\section{Nota de autor}

Esta investigación está financiada por el Grupo Consolidado de Investigación del Sistema Universitario Vasco IT1217-19 y el proyecto de investigación EDU2017-83949-P del subprograma estatal de Generación del Conocimiento del Ministerio de Economía, Industria y Competitividad. Su primera firmante es beneficiaria del Programa Predoctoral de Formación de Personal Investigador No Doctor del Departamento de Educación del Gobierno Vasco.

\section{BIBLIOGRAFÍA}

Aldridge, J, Fraser, B., Fozdar, F., Ala'i, K., Earnest, J. y Afari, E. (2015). Students' perceptions of school climate as determinants of wellbeing, resilience and identity. Improving Schools, 19(1), 5-26. https://doi.org/10.1177/1365480215612616

Alonso-Tapia, J., Quijada, A., Ruiz, M., Huertas, J.A., Ulate, M.A. y Biehl, M.L. (2020). A crosscultural study of the validity of a battery of questionnaires for assessing school climate quality. Psicología Educativa, 26(2), 109-119.

https://doi.org/10.5093/psed2020a2

Aron, A.M., Milicic, N. y Armijo, I. (2012). Clima social escolar: una escala de evaluación Escala de Clima Social Escolar, ECLIS-. Universitas Psychologica, 11(3), 803-813.

Bentler, P.M. (2006). EQS structural equations program manual. Encino, CA: Multivariate Software.

Bentler, P.M. y Wu, E.J. (2002). EQS for Windows user's guide. Encino, CA: Multivariate Software.

Bettencourt, L. (2004). Change-oriented organizational citizenship behaviors: The direct and moderating influence of goal orientation. Journal of Retailing, 8(3), 165-180. https://doi.org/10.1016/j.jretai.2003.12.001

Bondía, M., Martínez, I., Melendro, M. y Mora, R. (2019). Validación de un cuestionario sobre clima escolar para alumnado de Primaria. Journal for Educators, Teachers and Trainers, 10(1), 110-128.

Bosworth, K. y Judkins, M. (2014). Tapping into the power of school climate to prevent bullying: One application of schoolwide positive behavior interventions and supports. Theory Into Practice, 53(1), 300-307. https://doi.org/10.1080/00405841.2014.947224

Cohen, J., McCabe, E.M., Michelli, N.M. y Pickeral, T. (2009). School climate: Research, policy, teacher education and practice. Teachers College Record, 111(1), 180-213.

Daily, S.M., Mann, M.J., Kristjansson, A.L., Smith, M.L. y Zullig, K.J. (2019). School climate and academic achievement in middle and high school students. Journal of School Health, 89(3), 173-180. https://doi.org/10.1111/josh.12726

Daniels, H. (2016). Learning in cultures of social interaction. Revista de Investigación Educativa, 34(2), 315-328. http://dx.doi.org/10.6018/rie.34.2.252801

Durlak, J.A., Weissberg, R.P., Dymnicki, A.B., Taylor, R.D. y Schellinger, K.B. (2011). The impact of enhancing students' social and emotional learning: A meta-analysis of schoolbased universal interventions. Child Development, 82(1), 405-432. https://doi.org/10.1111/j.1467-8624.2010.01564.x

Escalante, N., Fernández-Zabala, A., Goñi, E e Izar-de-la-Fuente, I. (2020). Una nueva versión de la escala Percepción del Alumnado sobre el Clima Escolar (PACE) y análisis de sus propiedades psicométricas. Revista de Psicología y Educación, 15(2), 201-224. https://doi.org/10.23923/rpye2020.02.196

Escalante, N., Goñi, E., Fernández-Zabala, A. e Izar-de-la-Fuente, I. (2020). Diseño y estructura factorial del cuestionario Percepción del Alumnado sobre el Clima Escolar (PACE). European Journal of Education and Psychology, 13(1), 287-302. https://doi.org/10.30552/ejep.v13i1.294

Farina, K.A. (2019). Promoting a culture of bullying: Understanding the role of school climate and school sector. Journal of School Choice, 13(1), 94-120. 
https://doi.org/10.1080/15582159.2018.1526615

Gottfredson, G.D., Gottfredson, D.C., Payne, A.A. y Gottfredson, N.C. (2005). School climate predictors of school disorder: Results from a national study of delinquency prevention in schools. Journal of Research in Crime and Delinquency, 42(4), 412-444. https://doi.org/10.1177/0022427804271931

Hair, J., Babin, B. Anderson, R. y Black, W. (2018). Multivariate data analysis (8nd ed.). Hampshire, UK: Cengage Learnin, EMEA.

Herrera, K. y Ballesteros, R. (2014). El clima escolar como elemento fundamental de la convivencia en la escuela. Escenarios, 12(2), 7-18.

Hong, J.S., Espelage, D.L. y Lee, J.M. (2018). School climate and bullying prevention programs. En H. Shapiro (Ed.), The Wiley handbook on violence in education (pp. 359-374). Hoboken, NJ: John Wiley \& Sons. https://doi.org/10.1002/9781118966709.ch17

Hung, A.H., Luebbe, A.M. y Flaspohler, P.D. (2015). Measuring school climate: Factor analysis and relations to emotional problems, conduct problems, and victimization in middle school students. School Mental Health: A Multidisciplinary Research and Practice Journal, 7(2), 105-119. https://doi.org/10.1007/s12310-014-9131-y

IBM Corporation. (2017). IBM SPSS Statistics for Windows, version 25.0. Armonk, NY:IBM Corp.

Jia, Y., Way, N., Ling, G., Yoshikawa, H., Chen, X., Hughes, D., Ke, X. y Lu, Z. (2009). The influence of student perceptions of school climate on socioemotional and academic adjustment: A comparison of Chinese and American adolescents. Child Development, 80(5), 1514-1530. https://doi.org/10.1111/j.1467-8624.2009.01348.x

Kline, R.B. (2015). Principles and practice of structural equation modeling ( $4^{a}$ ed.). Nueva York, NY:Guilford Press.

López-González, L. y Bisquerra, R. (2013). Validación y análisis de una escala breve para evaluar el clima de clase en Educación Secundaria. ISEP Science, 5(1), 62- 77.

López-González, L. y Oriol, X. (2016). La relación entre competencia emocional, clima de aula y rendimiento académico en estudiantes de secundaria. Cultura y Educación, 28(1), 130-156. https://doi.org/10.1080/11356405.2015.1120448

Maxwell, S., Reynolds, K.J., Lee, E., Subasic, E. y Bromhead, D. (2017). The impact of school climate and school identification on academic achievement: Multilevel modeling with student and teacher data. Frontiers in Psychology, 8(1), 1-21. https://doi.org/10.3389/fpsyg.2017.02069

Muñoz, P.E., Casas, J.A., Del Rey, R., Ortega-Ruiz, R., Cerda, G. y Pérez, C. (2018). Validation and cross-cultural robustness of the School-wide Climate Scale (SCS) across Spanish and Chilean students. Studies in Educational Evaluation, 56(1), 182-188. https://doi.org/10.1016/j.stueduc.2018.01.002

National School Climate Center. (2014). School climate guidelines. Recuperado de https://www.schoolclimate.org/about/ourapproach/guidelines

Newland, L.A., DeCino, D.A., Mourlam, D.J. y Strous, G.A. (2019). School climate, emotions, and relationships: Children's experiences of well-being in the Midwestern U.S. International Journal of Emotional Education Special Issue, 11(1), 67-83.

Orpinas, P. y Raczynski, K. (2016). School climate associated with school dropout among tenth graders. Pensamiento Psicológico, 14(1), 9-20.

https://doi.org/10.11144/Javerianacali.PPSI14-1.scsd

Patton, G.C., Bond, L., Carlin, J.B., Thomas, L., Butler, H., Glover, S., Catalano, R. y Bowes, G. (2006). Promoting social inclusion in schools: A group-randomized trial of effects on student health risk behavior and well-being. American Journal of Public Health, 96(9), 1.582-1.587. https://doi.org/10.2105/AJPH.2004.047399

Quijada, A., Ruiz, M.A., Huertas, J.A. y Alonso-Tapia, J. (2020). Development and validation of the School Climate Questionnaire for Secondary and High School Teachers (SCQ-SHST). Anales de Psicología, 36(1), 155-165. https://doi.org/10.6018/analesps.341001 
Shindler, J., Jones, A., Williams, A.D., Taylor, C. y Cardenas, H. (2016). The school climate student achievement connection: If we want achievement gains, we need to begin by improving the climate. Journal of School Administration Research and Development, 1(1), 9-16.

Sun, L. y Royal, K. (2017). School climate in American secondary schools: A psychometric examination of PISA 2009 school climate scale. Journal of Curriculum and Teaching, 6(2), 6-12. https://doi.org/10.5430/jct.v6n2p6

Tabachnick, B.G. y Fidell, L.S. (2007). Using multivariate statistics ( $5^{a}$ ed.). New York, NY: Allyn and Bacon.

Varela, J.J., Sirlopú, D., Melipillán, R., Espelage, D., Green, J. y Guzmán, J. (2019). Exploring the influence school climate on the relationship between school violence and adolescent subjective wellbeing. Child Indicators Research, 12(6), 2095-2110. https://doi.org/10.1007/s12187-019-09631-9

Wang, M.T. y Degol, J.L. (2016). School climate: A review of the construct, measurement, and impact on student outcomes. Educational Psychology Review, 28(2), 315-352. 
ANEXO 1
ÍTEMS DEL PACE-33

\section{Items}

1. En este centro cuento con profesores/as a los que puedo contar los problemas que me surgen.

2. En este centro tenemos establecidas normas claras.

3. Los/las profesores/as esperan que los/las alumnos/as tengamos interés por aprender.

4. Mi centro está bien conservado.

5. Las normas se nos explican claramente a los/las alumnos/as.

6. Me siento a gusto hablando con mis compañeros/as de mis problemas.

7. Los/las profesores/as esperan que trabajemos duro.

8. En este centro cuento con compañeros/as a los que puedo contar los problemas que me surgen.

9. En este centro hay alumnos/as que se meten en peleas (empujones, patadas, etc.).

10. En esta clase hay un buen ambiente y una buena convivencia.

11. Los/las profesores/as están disponibles cuando necesito hablar con ellos/as.

12. Mi clase se mantiene limpia y ordenada.

13. En este centro hay alumnos/as que amenazan o insultan a otros/as alumnos/as.

14. En esta clase los/las alumnos/as nos cuidamos y ayudamos mutuamente.

15. Mis compañeros/as están disponibles cuando necesito hablar con ellos/as.

16. Los/las profesores/as esperan que los/las alumnos/as demos lo mejor de nosotros/as mismos/as.

17. En este centro está muy claro lo que está permitido y lo que está prohibido.

18. Los/las profesores/as plantean actividades originales.

19. En este centro hay alumnos/as que roban cosas.

20. Los/las alumnos/as, en general, nos llevamos bien entre nosotros/as.

21. El material con que trabajamos en clase me resulta interesante.

22. Es fácil hablar con los/las profesores/as.

23. Los/las alumnos/as conocemos las normas del centro. 


\section{Items}

24. Mi clase tiene una buena iluminación.

25. Mis compañeros/as me inspiran confianza para conversar sobre cosas personales.

26. Los/las profesores/as consiguen despertar nuestro interés por el trabajo en clase.

27. En esta clase los/las alumnos/as funcionamos como un equipo.

28. En este centro tengo profesores/as en los que puedo confiar.

29. Los/las profesores/as nos transmiten ganas de aprender.

30. Los demás espacios del centro están bien iluminados.

31. En este centro hay conflictos (peleas, amenazas, etc.).

32. Las actividades propuestas por el profesorado, en general, son atractivas.

33. Los/las profesores/as consiguen despertar nuestro interés por las asignaturas. 